\title{
SEOM clinical practice guideline: management and prevention of febrile neutropenia in adults with solid tumors (2018)
}

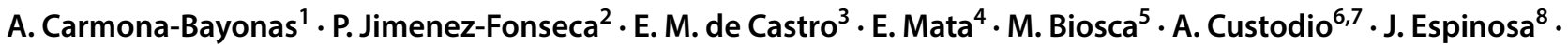 \\ E. G. Vázquez ${ }^{9,10} \cdot$ F. Henao ${ }^{11} \cdot$ F. Ayala de la Peña ${ }^{1}$ (i)
}

Received: 6 November 2018 / Accepted: 8 November 2018 / Published online: 23 November 2018

(C) The Author(s) 2018

\begin{abstract}
Febrile neutropenia (FN) is a common dose-limiting toxicity of chemotherapy, with a profound impact on the evolution of patients with cancer, due to the potential development of serious complications, mortality, delays, and decrease in treatment intensity. This article seeks to present an updated clinical guideline, with recommendations regarding the diagnosis, prevention, and treatment of febrile neutropenia in adults with solid tumors. The aspects covered include how to properly approach the risk of microbial resistances, epidemiological aspects, considerations about the initial empirical approach adapted to the risk, special situations, and prevention of complications. A decision-making algorithm is included for use in the emergency department based on a new, validated tool, the Clinical Index of Stable Febrile Neutropenia, which can be used in patients with solid tumors who appear stable in the initial phase of neutropenic infections, and can help detect those at high risk for complications in whom early discharge must be avoided.
\end{abstract}

Keywords CISNE score $\cdot$ Febrile neutropenia $\cdot$ SEOM $\cdot$ Clinical practice guideline

All of the authors have contributed equally to writing this manuscript.

F. Ayala de la Peña

frayala@um.es

A. Carmona-Bayonas

alberto.carmonabayonas@gmail.com

P. Jimenez-Fonseca

palucaji@hotmail.com

E. M. de Castro

evamdecastro@hotmail.com

E. Mata

elenamata28@hotmail.com

M. Biosca

mbiosca@vhebron.net

A. Custodio

anabcustodio@gmail.com

J. Espinosa

javiere@sescam.jccm.es

E. G. Vázquez

elisagarciavazquez@gmail.com

F. Henao

ferheca@gmail.com
1 Hematology and Medical Oncology Department, Hospital Universitario Morales Meseguer, IMIB, Avenida Marqués de los Vélez, 30008 Murcia, Spain

2 Medical Oncology Department, Hospital Universitario Central de Asturias, Oviedo, Spain

3 Medical Oncology Department, Hospital Universitario Marqués de Valdecilla, Santander, Spain

4 Medical Oncology Department, Complejo Hospitalario de Navarra, Pamplona, Spain

5 Medical Oncology Department, Hospital Vall D’Hebron, Barcelona, Spain

6 Medical Oncology Department, Hospital Universitario La Paz, Madrid, Spain

7 CIBERONC (CB16/12/00398), Madrid, Spain

8 Medical Oncology Department, Hospital General de Ciudad Real, Ciudad Real, Spain

9 Infectious Disease Department, Hospital Universitario Virgen de la Arrixaca, Murcia, Spain

10 Departamento de Medicina, Facultad de Medicina, Universidad de Murcia, Murcia, Spain

11 Medical Oncology Department, Complejo Hospitalario Regional Virgen Macarena, Seville, Spain 


\section{Abbreviations \\ ECOG-PS Eastern Cooperative Oncology Group perfor- mance status \\ NCI National Cancer Institute \\ COPD Chronic obstructive pulmonary disease}

\section{Introduction}

Febrile neutropenia $(\mathrm{FN})$ is defined as the appearance of fever (temperature $>38^{\circ} \mathrm{C}$ ) or clinical signs of sepsis, in a patient with a neutrophil count of $<500 / \mathrm{mm}^{3}$ or $<1000 / \mathrm{mm}^{3}$ prenadir $[1,2]$. It is a common iatrogenic complication, varying in incidence (2-50\%), depending on patient-related risk factors, neoplasm, type of chemotherapy, and genetic susceptibility [3-5]. While most patients experience mild episodes, the rate of serious complications (25-30\%) and mortality (9-12\%) remains elevated in high-risk groups [6]. The physiopathology of neutropenic sepsis covers the disruption of mucosal barriers, the architecture of the gastrointestinal lymphatic system, and the dissemination of bacterial inoculates throughout the bloodstream. Emergency $(<1 \mathrm{~h})$ initiation of broad-spectrum antimicrobial therapy is indispensable to preserve clinical stability.

In addition to its pharmacoeconomic impact [7, 8], neutropenia generates delays and dose reductions [9], which decreases the efficacy of chemotherapy [10, 11]. Primary prophylaxis of FN may be necessary for the most aggressive antineoplastic regimens or, in certain contexts, in subgroups of more vulnerable patients (e.g., the elderly) [12, 13].

The aim of this guideline, developed by the Spanish Society of Medical Oncology (SEOM, for its acronym in Spanish), is to set for the updated directives for the evidence-based diagnosis and treatment of FN in patients with solid tumors.

\section{Methods}

These guidelines have been developed by SEOM with the consensus of a panel of ten experts in the management of immunocompromised patients with solid tumors. The assignment of a level of evidence and grade of recommendation for each statement follows the Infectious Diseases Society of America-US Public Health Service Grading System for Ranking Recommendations in Clinical Guidelines. A full list of recommendations for febrile neutropenia is provided in Table 1.

\section{Etiology and antimicrobial susceptibility patterns}

In patients with solid tumors, FN has an identifiable clinical focus in $65 \%$ of the episodes; however, microbiological documentation is only possible in $20-30 \%$ of the cases and blood cultures are positive in 10-25\% [14, 15]. Etiology is usually bacterial, due to both Gram-negative bacilli, as well as Gram-positive cocci (approximate ratio of 3:2). Selective pressures that favor Gram-positive cocci infections in hematological patients (central venous catheter [CVC], prophylaxis with quinolones, mucositis grade $3-4$, etc.) are less intense in patients with solid cancer $[15,16]$. Anaerobic microorganisms and polymicrobial infections are uncommon, but can occur in special situations (e.g., abscesses, enteritis, etc.).

In recent years, there has been an increase in strains resistant to extended-spectrum $\beta$-lactamase (ESBL) or carbapenemases [17]. The risk of resistant microorganisms depends on previous colonization, invasive procedures, prior exposure to antibiotics, prior hospitalization, chronic comorbidity, and local pattern of resistances [II] [18-20]. The pattern of resistance recorded in Spain in 2015 for Gram positive is $10-25 \%$ to macrolides, in the case of pneumococcus; $25-50 \%$ to cloxacillin, in the case of Staphylococcus aureus; up to $90 \%$ for coagulase-negative staphylococci, and $1-5 \%$ resistance to vancomycin in the case of Enterococcus faecium [I, A] [21].

Invasive fungal infections (IFIs) are rare in patients with solid tumors $(<8 \%)$ [22]. Risk factors for IFI include prior use of antibiotics, having received multiple lines of chemotherapy, high-dose steroids (doses equivalent to or exceeding $20 \mathrm{mg}$ /day of prednisone for 4 weeks or more), extensive mucositis, CVC, and prolonged neutropenia ( $>7$ days). Candida albicans is responsible for most candidemias, with a recent increase in infections due to fluconazole-resistant species (e.g., Candida krusei and Candida glabrata). Seasonal respiratory viruses are common in contact with infected individuals, but reactivation of other latent viruses or relevant pathogens, common in the context of acute leukemia or bone marrow transplant, is unusual in the case of solid tumors [II, C].

\section{Initial evaluation and risk stratification}

The clinical history seeks to evaluate severity and clarify the clinical focus and epidemiological context (e.g., recent history of antibiotic use and the patient's microbiological record). To identify the infectious focus, a systematic physical examination is essential, targeting potential foci of infection (CVC, skin, and soft tissues, perineal and perianal area, respiratory system, oral cavity, pharynx, sinus pressure points, abdominal examination; as well as neurological and eye fundus examination, if symptoms such as scotomas or blurry vision are present).

Before administering empirical antibiotics, two sets of samples for blood cultures from different anatomic sites (if patient has $\mathrm{CVC}$, at least, one of the samples should be taken 
Table 1 General recommendations and levels of evidence

Diagnostic/treatment recommendation

Level, Grade

Initial clinical evaluation

The initial assessment should include the clinical history, physical examination, complete blood count, and basic biochemistry, and chest $\mathrm{Rx}$

Two pairs of blood cultures and microbiological specimens, as clinically indicated, should be obtained prior to initiating empirical III, A antibiotic therapy

Initial evaluation may include procalcitonin levels for the diagnosis of presumed bacterial infection and prognostic stratification

Consider a more comprehensive microbiological study in patients with clinical suspicion or history of specific infections, or with severe immunosuppression

Perform a computerized tomography of the chest in patients with clinically relevant respiratory symptoms and inconclusive chest $\mathrm{Rx}$, or in patients with persistent fever ( $72 \mathrm{~h}$ or more) and risk factors for complications

Initial prognostic evaluation

An initial prognostic evaluation according to clinical criteria is recommended to identify unstable patients or those at risk of severe complications

The application of the CISNE score is recommended in patients with apparent clinical stability and solid tumors on moderateintensity chemotherapy

\section{Initial empirical treatment}

Empirical antibiotic therapy should be initiated within the first hour of arrival at the ED (after sampling for cultures)

Initial parenteral antibiotherapy should include a beta-lactam with activity against Pseudomonas spp. (piperacillin/tazobactam, meropenem, imipenem-cilastatin, cefepime)

In high-risk patients meeting criteria for severity, initial parenteral antibiotherapy should include a beta-lactam with activity against Pseudomonas spp. (preferably a carbapenem) in combination with amikacin

In high-risk patients with suspicion of catheter-related infection or infection with a skin focus, pneumonia, or hemodynamic instability, it is recommended to associate vancomycin, linezolid (of choice if the focus is either pulmonary or cutaneous, but not recommended in catheter-related infections), or daptomycin (of choice in severe patients with quick SOFA $\geq 2$ points and suspicion of cutaneous or catheter focus) to initial antibiotherapy. Tigecycline should be used only as a last option

In high-risk patients with enterocolitis or perirrectal infection, metronidazole should be associated to a beta-lactam with antipseudomonal activity

In patients who are allergic to penicillin, we recommend aztreonam and vancomycin, with association of amikacin in situations of severity or Pseudomonas aeruginosa infection

In low-risk patients without prior prophylaxis with fluoroquinolones, after parenteral administration of the first doses, oral treatment can be undertaken with the combination of amoxicillin-clavulanic and levofloxacin or ciprofloxacin

In low-risk patients, oral fluoroquinolones in monotherapy should not be used

Therapeutic use of G-CSF is recommended in patients at high risk for infectious complications, with neutropenia $<100$ neutrophils $/ \mathrm{mm}^{3}$ or in the presence of risk factors (age $>65$, clinical instability, widespread infection, or severe complication)

Treatment in special situations

Antimicrobial treatment should be adapted to the isolates and patterns of resistance (detailed in Table 3)

II, A

Empirical antifungal treatment should be considered if the fever persists after 5-7 days and microbiological diagnoses are negative

If the fever persists after 5-7 days and microbiological diagnoses are negative, studies aimed at ruling out invasive fungal infection should be performed (determination of blood galactomannan titers, studies guided by clinical suspicion)

Hygiene-dietary measures

Prescription of a low-bacteria diet may be considered in patients with febrile neutropenia

Strict hand-washing protocols should be followed before and after contact with a patient with febrile neutropenia (FN)

Oral and skin hygiene should be optimized in patients with FN

Patients with resistant bacterial infections (methicillin-resistant Staphylococcus aureus, vancomycin-resistant Enterococcus spp, carbapenemase-resistant Enterococcus spp, or ESBL-producing Enterobacteriaceae, non-fermenting Gram-negative bacilli, such as Pseudomonas aeruginosa or Acznetobacter baumannii, with a multi-resistant pattern) should be isolated

Reverse isolation is recommended only in patients treated with chemotherapy associated with profound, prolonged neutropenia Follow-up and adjusting treatment

Daily clinical and analytical follow-up, with blood cultures every $48 \mathrm{~h}$ (and samples from other sites if applicable) in situations of persistent fever are recommended in patients with FN

Prevention and treatment of associated complications (thrombosis, cytopenias, mucositis) should be performed in patients with FN 
Table 1 (continued)

Diagnostic/treatment recommendation Level, Grade

Antibiotic treatment should be adapted to clinical suspicion, the infectious focus, and microbiological isolate in cases of persistence of fever and/or identification of the infectious focus

Specific empirical treatment against Gram-positive germs should be withdrawn if suspicion is not confirmed within $48 \mathrm{~h}$ of initia- $\quad$ II, $\mathrm{B}$ tion

Antibiotic coverage should be extended to Gram-negative bacilli, Gram-positive bacterium, and anaerobes in patients whose fever persists for more than $48 \mathrm{~h}$ associated with hemodynamic instability or clinical progression

Consultation with a specialist in infectious disease is recommended in bacteremias due to $S$. aureus, high-risk situations, multiresistant germs, atypical sites or treatments, or HIV infection

Empirical antibiotic treatment should be maintained for at least 7 days in low-risk FN without a clinical or microbiological focal site

Consider switching to oral antibiotic treatment in patients with low risk for FN without clinical or microbiological focal site with neutrophil count exceeding 500/mm3 and without fever for at least $72 \mathrm{~h}$

Antibiotic spectrum should be restricted according to infection focal site, severity, and the antibiogram of microorganisms identified as causing the infection after febrile neutropenia have resolved

Maintenance of antibiotherapy should be adjusted at least to the recommended duration for the underlying infection

Antibiotic treatment should last for at least 10-14 days in infections of the skin and soft tissue, pneumonias, and urinary tract infections

Hospital or ambulatory treatment

Patients meeting severity criteria should be treated as inpatients

Patients not meeting severity criteria and who appear to be clinically stable and present high-risk CISNE scores ( $\geq 3$ points) should be treated as inpatients

Patients not meeting severity criteria and who appear to be clinically stable and present high-risk CISNE scores (CISNE 3 or CISNE 2 with additional risk factors) should not be discharged from the hospital until clinical stability and negative results on blood culture are confirmed

Early discharge or ambulatory treatment with close follow-up may be considered in clinically stable patients not meeting criteria for severity, who exhibit low or intermediate risk scores on the CISNE model (CISNE 0-1) and who also meet the criteria for proximity, support, accessibility, and compliance with treatment and follow-up. However, CISNE does not specifically seek to select candidates for outpatient management, and standard hospitalization is also acceptable for these patients

In low-risk patients for whom ambulatory treatment has been decided on, the first dose of antibiotic should be administered via IV within the first hour and the patient should remain in observation at the hospital for at least $4 \mathrm{~h}$

Close supervision should be maintained of low-risk patients for whom ambulatory treatment has been decided on

In low-risk patients for whom ambulatory treatment has been decided on, detailed oral and written information should be provided regarding warning signs and symptoms, and contact data

Hospital readmission is recommended in low-risk patients for whom ambulatory treatment has been decided on who continue to have febrile neutropenia after $48-72 \mathrm{~h}$ of empirical treatment or/and who present new information about infection, oral intolerance, or for whom a change of antibiotic is indicated

Prevention of febrile neutropenia

Primary prophylaxis with filgrastim, pegfilgrastim, or biosimilar drugs is recommended when the probability of FN exceeds $20 \%$

The indication for primary prophylaxis with filgrastim, pegfilgrastim, or biosimilar drugs in patients with intermediate risk for neutropenic fever (10-20\%) should be individualized. The qualitative consequences of neutropenic infections, and not just their probabilities, must be considered

Secondary prophylaxis with filgrastim, pegfilgrastim, or biosimilar drugs is recommended in patients with a previous episode of FN in whom delaying or lowering the dose can negatively impact survival

As a general recommendation, antibiotic prophylaxis for FN should not be administered to patients undergoing mild-moderateintensity chemotherapy

In patients undergoing mild-moderate-intensity chemotherapy, antibiotic prophylaxis for FN may considered in exceptional cases with very high risk of infection

Prophylaxis should be assessed for patients with specific risks for $P$. jirovecii, tuberculosis, or hepatitis

II, A

II, A

II, B

III, B

Influenza vaccination is recommended in patients with solid tumors with active disease or/and undergoing chemotherapy

III, B

III, B

III, B

Vaccination against pneumococcus is recommended in patients with solid tumors with active disease or/and undergoing chemotherapy

I, A

II, A 
through the catheter) and microbiological samples should be taken, depending on the clinical orientation (e.g., urine, sputum, mucosal or skin lesions, feces, cerebrospinal fluid, urinary antigens for pneumococcus and/or Legionella spp., nasal swab for flu virus during flu season, etc.) [III, A]. A complete blood count and biochemistry is necessary with liver and kidney function tests, lactate, and a chest X-ray with two projections [III, B]. Procalcitonin (value $>0.5 \mathrm{ng}$ / $\mathrm{ml}$ ) may be more accurate and earlier than C-reactive protein (value $\geq 90 \mathrm{mg} / \mathrm{dl}$ ) to guide the diagnosis of bacteremia, and in predicting severity and FN complications, although its benefit is unclear with respect to the information provided by clinical criteria [II, B] [23].

In patients with pulmonary infiltrates and more profound immunosuppression (e.g., steroids, prolonged neutropenia, use of immunosuppressants, etc.), early bronchoscopy with bronchoalveolar lavage may be indicated. A full microbiological study (e.g., virus, fungi, atypical bacteria) is indicated in selected patients with clinical suspicion, previous infections or immunosuppression in addition to neutropenia [III, B]. In individuals with respiratory symptoms and inconclusive chest X-ray, or with persistent fever $\geq 72 \mathrm{~h}$ and risk factors for complications, a chest computed tomography is useful to detect incipient pneumonias, with $87 \%$ sensitivity and a negative predictive value of $88 \%$, surpassing chest Rx [24] [II, B]. Abdominal Rx has low sensitivity and specificity, and an abdominal CT is preferable in clinically suggestive cases and suspicion of abdominal focus (enterocolitis, typhlitis, etc.).

Severity is graded according to symptoms and signs, and risk assessment scores should only be applied when said signs and symptoms rule out clinical instability (see below) [III, B]. Most (3:1) individuals with FN and solid tumors display clinical stability in the first $3 \mathrm{~h}$ after diagnosis [25, 26]. High-risk patients or those who are potentially unstable are those with sepsis (defined as life-threatening acute organ dysfunction caused by the anomalous response to an infection, identifiable by an increase of 2 or more points on the quick SOFA scale), [27] septic shock, or severe documented infections (pneumonia, empyema, peritonitis, cellulitis $>4.5 \mathrm{~cm}$, suspected typhlitis, enteritis grade 3-4, appendicitis, cholecystitis, or other complicated abdominal infections, meningitis, encephalitis, catheter infections, endocarditis, and pyelonephritis) [28, 29]. In these unstable patients, mortality due to sepsis is $18.1 \%$ versus $2.7 \%$ of patients without risk factors [29]. Infections entailing greater mortality are chronic obstructive pulmonary disease (COPD) exacerbations, pneumonia, bacteremia (specially those caused by Gram-negative bacilli), and invasive fungal infections [6, 14]. Tumor progression and lung cancer are associated with a worse prognosis $[6,30,31]$. Furthermore, other toxicities (e.g., mucositis, enteritis, thrombopenia) or coexisting complications (thromboembolic disease, need for transfusion, etc.) affect decision-making [25, 26, 32]. Table 2 is a complete list of clinical characteristics that define an episode of $\mathrm{FN}$ as being high risk.

Clinical assessment in subjects with inflammatory responses decreased by immunosuppression is complex, and some seemingly stable episodes remain at potential risk of complications, despite not showing evident criteria of severity at the onset of the FN episode. In three contemporary series, the frequency of unexpected serious complications in apparently stable individuals in the first $3 \mathrm{~h}$ was $7.3-18.6 \%$ [25, 29]. The Clinical Index of Stable Febrile Neutropenia (CISNE) (Table 2) was developed and validated for the Western population to predict serious complications (shock, acute organ failure, arrhythmia, major bleeding, delirium, disseminated intravascular coagulation, acute abdomen), in the subgroup of patients with solid tumors who have undergone mild-moderateintensity chemotherapy and who appear to be clinically stable at the onset of the episodes [II, B] [14, 25, 26, 29]. Its application requires having previously ruled out hemodynamic instability or clinical risk of complications by means of vital signs, anamnesis, physical examination, and

Table 2 Risk factors for complications that contraindicate ambulatory management

\begin{tabular}{ll}
\hline Organ or system & Risk factor \\
\hline Vascular & $\begin{array}{c}\text { Syncope, systolic blood pressure }<90 \mathrm{mmHg} \text {, hypertensive crisis, arrhythmias, heart failure, clinically relevant bleeding, } \\
\text { angina pectoris }\end{array}$ \\
Hematological & Other clinically relevant cytopenias (platelets $<50,000 / \mu \mathrm{L}, \mathrm{Hb}<8 \mathrm{~g} / \mathrm{dL}$ ), thromboembolic disease \\
Gastrointestinal & Oral intolerance, vomiting, diarrhea, pain abdominal, jaundice, alteration of liver function tests \\
Infectious & Sepsis or severe focus (pneumonia, extensive cellulitis, bacteremia, catheter, pyelonephritis, meningitis, cholecystitis, and \\
& other surgical infections), allergy to antibiotics, recent use of antibiotics \\
Neurological & Presence of focal neurological symptoms, suspicion of meningitis, acute confusional syndrome \\
Pulmonary & Abscesses, pneumothorax, pleural effusion, acute respiratory failure, pulmonary infiltrates, or cavitary nodules \\
Others & Acute kidney failure, dehydration, electrolyte alterations, other alterations of vital signs, other complications considered \\
& severe, pregnancy, fractures
\end{tabular}


Table 3 The CISNE score

\begin{tabular}{lll}
\hline Characteristics & Weight (points) \\
\hline ECOG-PS $\geq 2$ & 2 & \\
Stress-induced hyperglycemia & 2 & \\
Chronic obstructive pulmonary disease & 1 & \\
Chronic cardiovascular disease & 1 & \\
Mucositis NCI grade $\geq 2$ & 1 & \\
Monocytes $<200$ per $\mu \mathrm{L}$ & 1 & \\
\hline CISNE prognostic categories & & Score $^{\mathrm{a}}$ \\
\hline Low risk & & 0 \\
Intermediate risk & & $1-2$ \\
High risk & $3-8$ \\
\hline
\end{tabular}

${ }^{\mathrm{a}} \mathrm{A}$ CISNE score $\geq 3$, equals $\geq 116$ points on the nomogram, or a risk of $\geq 13 \%$, on the online calculator

laboratory data (Table 3). CISNE should never be used in hematological patients or individuals with lymphoma, those who are unstable, with serious infections, or who have contraindications for ambulatory treatment for other reasons. There is no experience regarding its application to select patients at low risk for direct ambulatory treatment. Online calculators for CISNE are available and provide a categorized (https://www.mdcalc.com/clinical-index-stabl e-febrile-neutropenia-cisne) or continuous estimate of risk (http://www.iricom.es/prognostictools/cisne/inicio.aspx).

The usefulness of the Multinational Association of Supportive Care in Cancer (MASCC) is unknown in predicting complications in patients deemed stable and one must be cognizant of its methodological limitations (coincidence of hypotension as the main predictor and endpoint, heterogeneous population with hematological neoplasms, etc.) [1], and of its low sensitivity in stable patients (34.8-36\%) [23, 24, 26, 31-34].

\section{Initial empirical treatment}

Empirical treatment should be selected on the basis of the type and severity of the infection, and the probability of antibiotic-resistant microorganisms being involved in its etiology. The first dose of empirical antibiotic therapy should be initiated within the first hour and after taking samples for culture; hospitals should, therefore, adopt suitable logistical measures [II, A].

For all patients, intravenous administration of a $\beta$-lactam with antipseudomonal activity but that conserves activity against Gram positive is recommended (piperacillin-tazobactam $4.5 \mathrm{~g} / 6 \mathrm{~h}$; meropenem $1 \mathrm{~g} / 8 \mathrm{~h}$; imipenem-cilastatin $500 \mathrm{mg} / 6 \mathrm{~h}$; cefepime $2 \mathrm{~g} / 8 \mathrm{~h}$ ) [I, A]. This regimen must be modified depending on the patient's prognosis and characteristics, as indicated below: (a) High-risk patients: parenteral $\beta$-lactam with antipseudomonal activity should be combined with another agent chosen on the basis of the clinical situation:

- In the event that piperacillin-tazobactam is used, amikacin 15-20 mg/kg/day intravenous [IV] should be associated, or substitute the $\beta$-lactam for imipenem or meropenem due to the growing problem of Pseudomonas spp. infections, particularly in oncological patients, and ESBL-producing Gram-negative bacilli [II, A].

- In situations of severity (e.g., quick SOFA $\geq 2$ points), associate amikacin $15-20 \mathrm{mg} / \mathrm{kg} /$ day IV (higher doses may be appropriate depending on isolation and pharmacokinetic data) [II, A] to the $\beta$-lactam (preferably a carbapenem).

- When a catheter-related infection, skin focus, or pneumonia is suspected or if the patient presents hemodynamic instability, associate vancomycin (15-20 mg/kg/8-12 h IV), linezolid (of choice if the focus is pulmonary or cutaneous, but not recommended in catheter-related infections), or daptomycin (of choice in severe patients with quick $S O F A \geq 2$ points and suspicion of cutaneous or catheter focus) to the $\beta$-lactam [II, A]. Tigecycline (50-100 mg/12 h IV after an initial dose of 100-200 mg) is an alternative that may be used as a last option given the increase in all-cause mortality observed in a metaanalysis.

- In case of enterocolitis (typhlitis) or perirrectal infection, the previously mentioned $\beta$-lactams are active; however, given the risk of possible resistance, the recommendation is that parenteral metronidazole $500 \mathrm{mg} / 6 \mathrm{~h}$ be associated [II, A].

- In the event of prior carbapenem-resistant Pseudomonas aeruginosa colonization or of high risk due to high incidence of said strains in the patient's setting and severity, assess the use of ceftolozanetazobactam or ceftazidime-avibactam (unless resistance is already known) as $\beta$-lactam or associate colistimethate.

(b) In patients who are allergic to penicillin, the $\beta$-lactam must be substituted and aztreonam $1 \mathrm{~g} / 8 \mathrm{~h}$ in association with vancomycin (or linezolid, tigecycline or daptomycin; see previous paragraph) used in its place, assessing the addition of metronidazole and/or amikacin, according to previous indications [II, B]. Consider the possibility that Pseudomonas aeruginosa may be resistant to aztreonam; therefore, in the previously mentioned situations it is wise to always associate amikacin, and later, with the data from the antibiogram in hand, assess the patient's desensitization to the 
$\beta$-lactam of choice according to the microbiology data and focus of infection [II, B].

(c) Low-risk patients for infection by resistant microorganisms and for complications: after the first intravenous doses, if the patient has not received prophylaxis with fluoroquinolones, oral treatment (always in combination) is an alternative that is deemed effective:

- Amoxicillin-clavulanic $875 / 175 \mathrm{mg} / 8 \mathrm{~h}$ or 1000/62.5 mg 2 comp/12 h (per os) (clindamycin $300 \mathrm{mg} / 8 \mathrm{~h}$ in case of allergy to penicillin) + Levofloxacin $750 \mathrm{mg} /$ day P.O. or ciprofloxacin $750 \mathrm{mg} / 12 \mathrm{~h}$ P.O. [II, B]. In patients who are allergic to $\beta$-lactams, there is no good oral treatment option. In these cases, the initial treatment should be intravenous; hospital discharge is not recommended until microbiological documentation is available or after $48-72 \mathrm{~h}$ of clinical stability.

In our setting, in which enterobacteria resistant to quinolones and non-fermenting bacteria are high (up to 50\%), initial treatment with fluoroquinolones in monotherapy is not recommended, not even in low-risk patients [II, D]. In general, antibiotic doses must be adjusted whenever necessary for the patient's kidney function.

Therapeutic use of granulocyte-colony stimulating factor (G-CSF) is recommended in episodes of FN in patients at high risk for infectious complications, with profound neutropenia $(<100$ neutrophils $/ \mathrm{mm} 3)$, or in the presence of adverse prognostic factors, such as age $>65$ years, unstable patients, with serious complications or widespread infections [I, A] [35, 36]. Their routine use is not recommended, since, although it decreases duration and hospital stay, it does not enhance survival $[35,36]$.

\section{Treatment in special situations}

For infections due to certain resistant microorganisms or in special situations, there are specific recommendations to modify the general regimen, collected in Table 4 .

As regards fungal infections, empirical antifungal treatment must be initially considered ("preemptive therapy") if, after 5-7 days of antibiotic treatment, the patient continues to be febrile and the initial microbiological studies are negative [I, A]; the most appropriate time depends on the clinical setting and the presence of risk factors for invasive fungal infection (IFI) (prolonged neutropenia, immunosuppressant treatment, corticosteroids, etc.). A galactomannan titer in blood of $>0.5$ (optical density index) on two consecutive determinations, or $>0.7$ in a single determination, is valuable for the diagnosis of invasive aspergillosis. Furthermore, the IFI must be characterized by means of targeted studies based on clinical suspicion (CT; ear, nose and throat exploration, lung puncture aspirate, biopsy of skin lesions, etc.) [III, A]. The choice of antifungal drug (echinocandin, voriconazole, or liposomal amphotericin B) is conditioned by the prior use of azoles, clinical suspicion, and results of the galactomannan test. Echinocandins would only be of choice in cases of suspected Candida spp. infection (patients with widespread mucositis, patients with CVC and/or parenteral nutrition); in the event that Aspergillus spp. infection is suspected (lung nodules, positive galactomannan test), voriconazole is the drug of choice [37]. At centers with a high incidence of Mucorales, initiating liposomal amphotericin B treatment must be evaluated.

\section{Hygiene-dietary considerations}

Patients with FN are sometimes prescribed a diet low in bacteria, without having confirmed that this diet lowers the risk of infection or mortality [III, C] [38]. Hand hygiene is the most effective procedure for preventing hospital acquired infections; all personnel should, therefore, follow hand-washing protocols before entering and after leaving the room of a neutropenic patient, as well as the "5 moments" approach of hand-washing as per the guidelines of the World Health Organization [I, A] [39]. General protection measures should be adopted (e.g., gloves, gowns, and/or masks) [40] and the contact isolation measures established in each for specific isolations: methicillin-resistant Staphylococcus aureus, vancomycin-resistant Enterococcus spp, carbapenemase- or ESBL-producing Enterobacteriaceae, nonfermenting Gram-negative bacilli, such as Pseudomonas aeruginosa or Acinetobacter baumannii, with a pattern of multi-resistance [II, A]. Moreover, patients should optimize their oral and skin hygiene [II, A]. In patients with solid tumors, reverse isolation is indicated only for patients being treated with chemotherapy schedules that produce profound and prolonged neutropenia [II, A] [40].

\section{Follow-up and treatment adjustment}

During follow-up of a patient with FN, a physical examination must be performed and their general daily status must be evaluated to rule out the appearance of new infectious foci. Vital signs must be taken every $8 \mathrm{~h}$. Hemogram and biochemistry with daily kidney and liver function tests must be carried out, and blood cultures every $48 \mathrm{~h}$ in patients with persistent fever, in addition to sampling of other foci that may appear. Supervision, prevention, and treatment of other complications (thrombosis, anemia, thrombopenia, intestinal dysbacteriosis, mucositis, etc.) are needed [III, A].

The adjustments made to the antibiotic regimen will depend on the patient's evolution, the infectious focus, and the sensitivity profile of the microbiological isolations. Likewise, if 


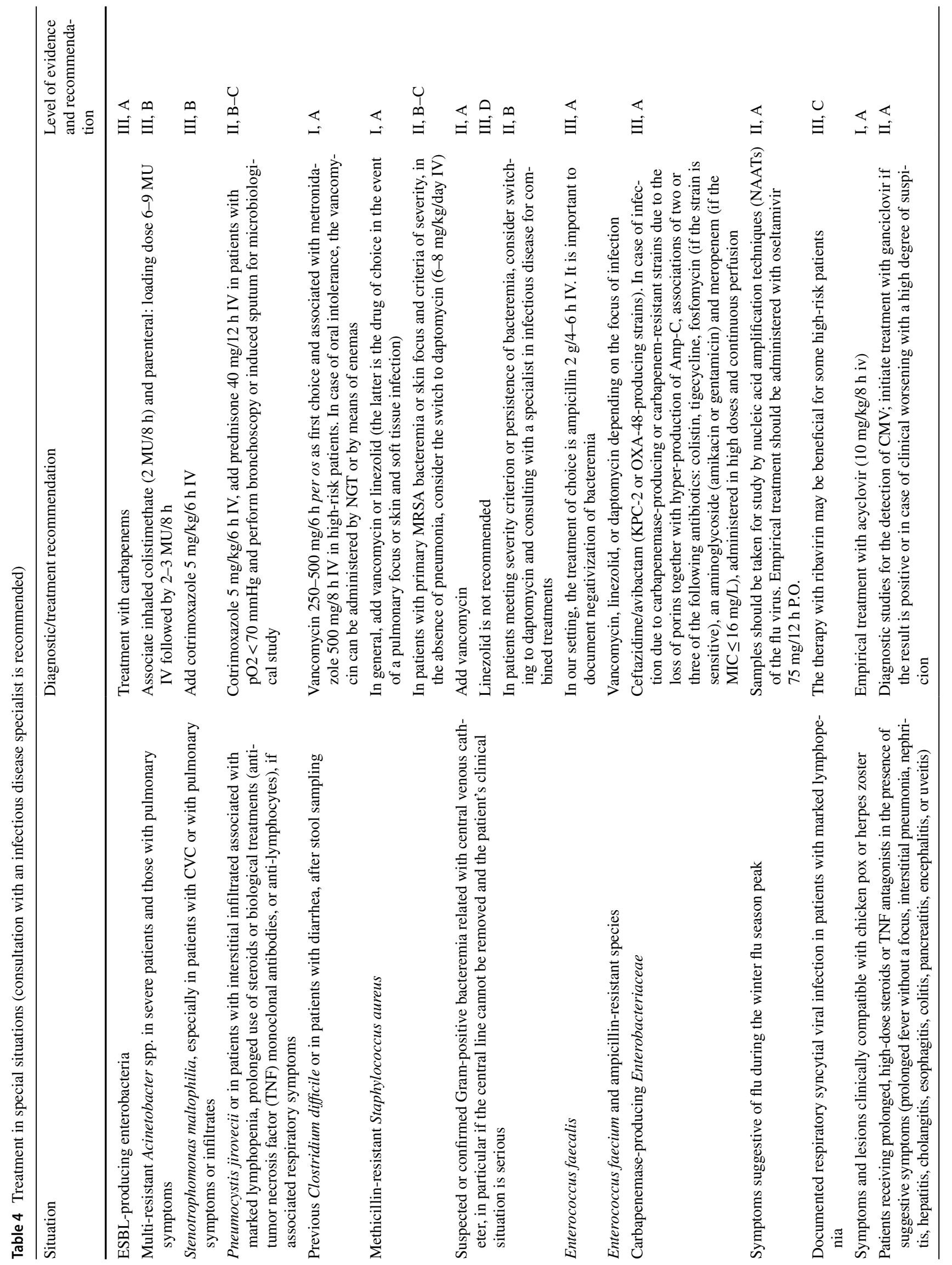


the fever persists and the focus of infection is identified, the antibiotherapy should be modified based on a specific suspicion or on the microbiological isolation [I, A]. In the case of having initiated specific empirical treatment against Grampositive bacilli (e.g., glycopeptide), it must be withdrawn $48 \mathrm{~h}$ after its initiation if the initial suspicion is not confirmed [II, B]. In patients who have been febrile $>48 \mathrm{~h}$ without clinical worsening, a wait and see approach can be adopted, actively searching for the clinical source of infection by means of serial blood cultures, stool cultures, and imaging studies depending on the clinical suspicion. Similarly, peripheral venous catheters should also be rotated periodically and non-infectious causes of fever should also be assessed. In patients with persistent fever $>48 \mathrm{~h}$ who also present hemodynamic instability or clinical progression, the empirical antimicrobial spectrum should be expanded to cover resistant Gram-negative bacilli, Grampositive bacteria, and anaerobes [I, A]. Antifungal medications may be necessary in patients who meet criteria of severity and have persistent fever $>4-7$ days (see treatment in special situations, above). In cases that so require and depending on the patient's options for recovery and the cancer's prognosis, the intensive care unit will be contacted [41]. The isolation of pathogens resistant to one or more of the components of the initial antibiotic therapy entails the need to adjust it (Table 4). A specialist in infectious disease or experienced clinician should be consulted when faced with patients with poor evolution, $S$. aureus bacteremia, persistent bacteremia, atypical microorganisms or resistances, highly severity infectious syndromes (CNS involvement, endocarditis, etc.), the need for antimicrobials with which the attending oncologist is relatively unfamiliar (Table 3), or HIV infection [III, C].

Empirical antibiotic treatment should last for a minimum of 7 days in low-risk FN without an identified clinical or microbiological focal site. When the neutrophil count exceed 500 cells $/ \mathrm{mm} 3$ and the patient has been fever free for $72 \mathrm{~h}$, oral administration can commence (as per the same considerations as in the previous section) [III, A]. In FN with clinical or microbiologically documented infection, said treatment should be followed in accordance with the recommended duration for the underlying infectious process [III, B]. In the specific case of skin and soft tissue infections, pneumonias, and urinary tract infections, antibiotic treatment should be followed for 10-14 days in most cases [II, B]. In the event of microbiological documentation, the antibiotic spectrum can be decreased depending on the focus and severity of the infection, and the antibiogram of the microorganisms identified as the cause of the infectious disease [II, A].

\section{Inpatient treatment, early discharge, and outpatient treatment}

Hospital treatment should be considered standard, whereas home treatment or early discharge is an alternative in selected, low-risk cases [4], defined as those that have no severity criteria [II, A] (Table 2). The CISNE model should only be used in patients in a situation of apparent clinical stability, after having ruled out clinical, epidemiological, or analytic criteria of severity, with the aim of avoiding early discharge of patients at potential risk for complications or severe sepsis.

CISNE does not seek to select patients for outpatient management, but to safeguard them from inappropriate discharge until favorable evolution is confirmed. Figure 1 illustrates the decision-making algorithms that make up the CISNE score.

In particular, stable patients who are qualified as high risk according to CISNE (score $\geq 3$, its equivalent $\geq 116$ points on the nomogram, or risk for complications $\geq 13 \%$ on the on-line calculator) should be treated in the hospital and should not be discharged until observation has demonstrated that the apparent stability is real and blood cultures have proven negative [II, B] [26, 29, 42]. In patients with a CISNE score of 2, additional clinical or microbiological risk factors should be considered that might also contradict ambulatory management.

In patients who are at low risk (score CISNE 0-1), admission to hospital with early discharge, standard hospital admission or ambulatory treatment with close followup can be considered, depending on each episode's clinical presentation [III, B]. In any case, given the potential risk of unexpected complications even in the lowest risk group (4.2\% of the patients with $0-57$ points on the CISNE nomogram) [29], for a patient with FN to be treated at home, all the following criteria must also be met: reside within $1 \mathrm{~h}$ or less than $50 \mathrm{~km}$ from the hospital; have a means of transportation available, access to a telephone, and good family support in the home for $24 \mathrm{~h}$; have the approval of their referring oncologist/clinician; foresee good compliance with regular doctor's visits; expected compliance with the oral administration of medications; and no prior history of non-compliance with treatment [III, B] [32]. After being identified as low risk and initiating empirical antibiotic therapy and prior to discharge, patients must remain in observation for at least $4 \mathrm{~h}$ to verify their stability and tolerance to treatment [III, C] [32]. If the decision is made to discharge the patient, close follow-up is mandatory with clinical reevaluation and hemogram $48 \mathrm{~h}$ later, and daily phone contact for a minimum of 3 days to check that the fever has resolved. It is important to properly inform the patient (orally and in writing) about the 
detection of warning signs and symptoms, as well as how to contact the healthcare personnel $24 \mathrm{~h} /$ day [III, B] [29, $32,43]$. Hospital admission must be contemplated when fever or neutropenia persists after 2-3 days of treatment with empirical antibiotic therapy, new signs or symptoms of infection, oral intolerance, or need to change antibiotherapy [III, B] [32].

\section{Prevention of febrile neutropenia: prophylaxis with G-CSF}

The risk of developing FN must be evaluated on a case-bycase basis at the beginning of each cycle of chemotherapy [44]. Primary prophylaxis with G-CSF is recommended when the likelihood of developing FN is $>20 \%[2,11,45$, 46], having demonstrated a decrease in incidence, duration, and severity, without significantly affecting tumor response or survival [I, A] [35, 47]. In patients with an intermediate level of risk (10-20\%), the indication of prophylaxis with G-CSF must be individualized depending on the characteristics that increase the risk of FN (age $>65$ years, liver or kidney dysfunction, widespread bone marrow involvement, recent extensive surgery, persistent neutropenia, or prior episodes of FN) [II, A] [2, 11, 45, 46]. In special circumstances, regardless of frequency, individuals may also be considered eligible for primary prophylaxis if the consequences of the neutropenic episode are foreseen to be more severe.

The recommended dose of filgastrim is $5 \mu \mathrm{g} / \mathrm{kg} / \mathrm{day}$ subcutaneously administered $24-72 \mathrm{~h}$ after administration of chemotherapy until achieving a sufficient, stable postnadir neutrophil count [I, A]. Pegfilgastrim (a single dose of $100 \mu \mathrm{g} / \mathrm{kg}$ or $6 \mathrm{mg}$ ) can be more effective in different contexts and approved biosimilar drugs can also be considered $[2,45]$.

In patients who have experienced a prior episode of $\mathrm{FN}$ or dose-limiting neutropenia [48], the use of secondary prophylaxis with G-CSF can be considered individually if the dose reductions or delay of antineoplastic treatments can negatively affect prognosis [I, A] [2, 11, 45, 46].

\section{Prevention of febrile neutropenia: antimicrobials and vaccination}

In patients with solid tumors treated with chemotherapy of mild-moderate intensity, the use of prophylaxis with oral antibiotics to prevent episodes of FN [I, E] is not recommended, except for exceptional cases of patients with very high risk of infection in whom antineoplastic therapy cannot be delayed [III, B].

In patients with specific risks, prophylaxis against $P n e u-$ mocystis jirovecii, tuberculosis [49], or viral hepatitis should

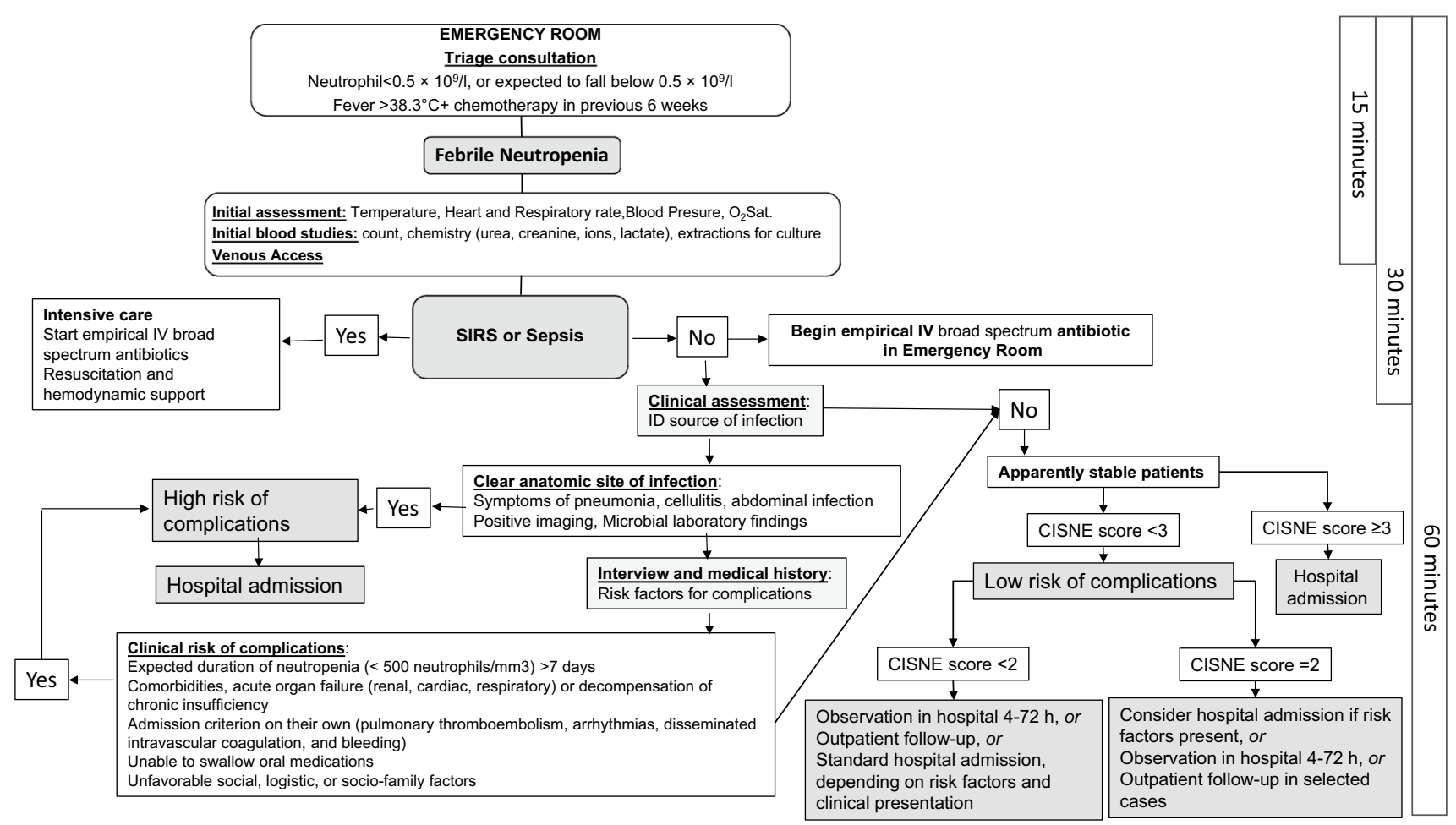

Fig. 1 Decision-making algorithm in febrile neutropenia 
be assessed according to recommendations found in other clinical practice guidelines [II, A].

Patients with active solid tumors and those receiving chemotherapy should be vaccinated every year against flu [II, A] and against pneumococcus [III, A] in accordance with guidelines for immunodepressed patients. Other vaccinations may be advisable depending on the kind of chemotherapy, the patient's clinical status, or a specific indication [43].

\section{Compliance with ethical standards}

Conflict of interest ACB have received speaker and travel grants from IPSEN, Servier, and Roche, outside the submitted work. PJF has nothing to disclose. EMC has nothing to disclose. EM has nothing to disclose. MB reports personal fees from Leo Pharma, Rovi and Sanofi, outside the submitted work. AC has nothing to disclose. JE reports personal fees from MSD, Novartis, Pfizer, BMS, Astra Zeneca and Roche, outside the submitted work. EGV has nothing to disclose. FH has nothing to disclose. FAP reports grants from Roche and Celgene, and other financial support from Roche, Celgene, Eisai, AstraZeneca, Pfizer, Pierre Fabre and Novartis, outside the submitted work.

Ethical standards The current study has been performed in accordance with the ethical standards laid down in the 1964 Declaration of Helsinki and its later amendments. This article does not contain any studies with human participants or animals performed by any of the authors.

Informed consent For this type of study, no informed consent is required.

Open Access This article is distributed under the terms of the Creative Commons Attribution 4.0 International License (http://creativeco mmons.org/licenses/by/4.0/), which permits unrestricted use, distribution, and reproduction in any medium, provided you give appropriate credit to the original author(s) and the source, provide a link to the Creative Commons license, and indicate if changes were made.

\section{References}

1. Klastersky J, Paesmans M, Rubenstein EB, Boyer M, Elting L, Feld R, et al. The multinational association for supportive care in cancer risk index: a multinational scoring system for identifying low-risk febrile neutropenic cancer patients. J Clin Oncol. 2000;18(16):3038-51.

2. Klastersky J, de Naurois J, Rolston K, Rapoport B, Maschmeyer G, Aapro M, et al. Management of febrile neutropaenia: ESMO Clinical Practice Guidelines. Ann Oncol. 2016;27(5):v111-8.

3. Weycker D, Barron R, Kartashov A, Legg J, Lyman GH. Incidence, treatment, and consequences of chemotherapy-induced febrile neutropenia in the inpatient and outpatient settings. J Oncol Pharm Pract. 2014;20(3):190-8.

4. Taplitz RA, Kennedy EB, Flowers CR. Outpatient management of fever and neutropenia in adults treated for malignancy: American Society of Clinical Oncology and Infectious Diseases Society of America Clinical Practice Guideline update summary. J Oncol Pract. 2018;14:250-5.

5. McCune JS, Sullivan SD, Blough DK, Clarke L, McDermott C, Malin J, et al. Colony-stimulating factor use and impact on febrile neutropenia among patients with newly diagnosed breast, colorectal, or non-small cell lung cancer who were receiving chemotherapy. Pharmacotherapy. 2012;32(1):7-19.

6. Kuderer NM, Dale DC, Crawford J, Cosler LE, Lyman GH. Mortality, morbidity, and cost associated with febrile neutropenia in adult cancer patients. Cancer. 2006;106(10):2258-66.

7. Mayordomo JI, López A, Viñolas N, Castellanos J, Pernas S, Domingo Alonso J, et al. Retrospective cost analysis of management of febrile neutropenia in cancer patients in Spain. Curr Med Res Opin. 2009;25(10):2533-42.

8. Schilling MB, Parks C, Deeter RG. Costs and outcomes associated with hospitalized cancer patients with neutropenic complications: a retrospective study. Exp Ther Med. 2011;2(5):859-66.

9. Pettengell R, Schwenkglenks M, Leonard R, Bosly A, Paridaens $\mathrm{R}$, Constenla M, et al. Neutropenia occurrence and predictors of reduced chemotherapy delivery: results from the INC-EU prospective observational European neutropenia study. Support Care Cancer. 2008;16(11):1299-309.

10. Lyman GH, Michels SL, Reynolds MW, Barron R, Tomic KS, Yu J. Risk of mortality in patients with cancer who experience febrile neutropenia. Cancer. 2010;116(23):5555-63.

11. Aapro MS, Bohlius J, Cameron DA, Lago LD, Donnelly JP, Kearney N, et al. 2010 update of EORTC guidelines for the use of granulocyte-colony stimulating factor to reduce the incidence of chemotherapy-induced febrile neutropenia in adult patients with lymphoproliferative disorders and solid tumours. Eur J Cancer. 2011;47(1):8-32.

12. Balducci L, Al-Halawani H, Charu V, Tam J, Shahin S, Dreiling $\mathrm{L}$, et al. Elderly cancer patients receiving chemotherapy benefit from first-cycle pegfilgrastim. Oncologist. 2007;12(12):1416-24.

13. von Minckwitz G, Kümmel S, du Bois A, Eiermann W, Eidtmann $\mathrm{H}$, Gerber B, et al. Pegfilgrastim \pm ciprofloxacin for primary prophylaxis with TAC (docetaxel/doxorubicin/cyclophosphamide) chemotherapy for breast cancer. Results from the GEPARTRIO study. Ann Oncol. 2008;19(2):292-8.

14. Carmona-Bayonas A, Jiménez-Fonseca P, Virizuela J, Antonio $\mathrm{M}$, Font $\mathrm{C}$, Biosca $\mathrm{M}$, et al. Performance of the clinical index of stable febrile neutropenia (CISNE) in different types of infections and tumors. Clin Transl Oncol. 2016;19(3):1-10.

15. Carmona-Bayonas A, Fonseca PJ, Font C, Martínez-García J, Torrella F, Urrego E, et al. Bacteremia in adult cancer patients with apparently stable febrile neutropenia: data from a cohort of 692 consecutive episodes from a single institution. J Community Support Oncol. 2014;12(9):312-20.

16. Viscoli C. Management of infection in cancer patients: studies of the EORTC International Antimicrobial Therapy Group (IATG). Eur J Cancer. 2002;38:82-7.

17. Trecarichi EM, Tumbarello M. Antimicrobial-resistant Gramnegative bacteria in febrile neutropenic patients with cancer: current epidemiology and clinical impact. Curr Opin Infect Dis. 2014;27(2):200-10.

18. Ben-Ami R, Rodríguez-Baño J, Arslan H, Pitout JDD, Quentin C, Calbo ES, et al. A multinational survey of risk factors for infection with extended-spectrum $\beta$-lactamase-producing Enterobacteriaceae in nonhospitalized patients. Clin Infect Dis. 2009;49(5):682-90.

19. Ram R, Farbman L, Leibovici L, Raanani P, Yeshurun M, Vidal L, et al. Characteristics of initial compared with subsequent bacterial infections among hospitalised haemato-oncological patients. Int J Antimicrob Agents. 2012;40(2):123-6.

20. Furuno JP, McGregor JC, Harris AD, Johnson JA, Johnson JK, Langenberg $\mathrm{P}$, et al. Identifying groups at high risk for carriage of antibiotic-resistant bacteria. Arch Intern Med. 2006;166(5):580-5. 
21. EARS-Net. Antimicrobial resistance surveillance in Europe 2015. Annual report of the European Antimicrobial Resistance Surveillance Network (EARS-Net); 2015.

22. Mensa J, Gatell JM, García-Sánchez JE, Marco E, Letang E, López-Suñé F. Guía de terapéutica antimicrobiana. Barcelona: Antares; 2017.

23. Carmona-Bayonas A, Gómez J, González-Billalabeitia E, Canteras M, Navarrete A, Gonzálvez ML, et al. Prognostic evaluation of febrile neutropenia in apparently stable adult cancer patients. Br J Cancer. 2011;105(5):612-7.

24. Heussel CP, Kauczor H-U, Ullmann AJ. Pneumonia in neutropenic patients. Eur Radiol. 2004;14(2):256-71.

25. Carmona-Bayonas A, Gómez J, González-Billalabeitia E, Canteras M, Navarrete A, Gonzálvez ML, et al. Prognostic evaluation of febrile neutropenia in apparently stable adult cancer patients. Br J Cancer. 2011;105(5):612.

26. Carmona-Bayonas A, Jiménez-Fonseca P, Echaburu JV, Antonio $\mathrm{M}$, Font $\mathrm{C}$, Biosca $\mathrm{M}$, et al. Prediction of serious complications in patients with seemingly stable febrile neutropenia: validation of the clinical index of stable febrile neutropenia in a prospective cohort of patients from the FINITE study. J Clin Oncol. 2015;33(5):465.

27. Singer M, Deutschman CS, Seymour CW, Shankar-Hari M, Annane D, Bauer M, et al. The Third International consensus definitions for sepsis and septic shock (Sepsis-3). JAMA. 2016;315(8):801-10.

28. Bone RC, Balk RA, Cerra FB, Dellinger RP, Fein AM, Knaus WA, et al. Definitions for sepsis and organ failure and guidelines for the use of innovative therapies in sepsis. Chest. 1992;101(6):1644-55.

29. Fonseca PJ, Carmona-Bayonas A, García IM, Marcos R, Castañón $\mathrm{E}$, Antonio M, et al. A nomogram for predicting complications in patients with solid tumours and seemingly stable febrile neutropenia. Br J Cancer. 2016;114(11):1191-8.

30. Caggiano V, Weiss RV, Rickert TS, Linde-Zwirble WT. Incidence, cost, and mortality of neutropenia hospitalization associated with chemotherapy. Cancer. 2005;103(9):1916-24.

31. Talcott JA, Siegel RD, Finberg R, Goldman L. Risk assessment in cancer patients with fever and neutropenia: a prospective, two-center validation of a prediction rule. J Clin Oncol. 1992;10(2):316-22.

32. Flowers CR, Seidenfeld J, Bow EJ, Karten C, Gleason C, Hawley DK, et al. Antimicrobial prophylaxis and outpatient management of fever and neutropenia in adults treated for malignancy: American Society of Clinical Oncology Clinical Practice Guideline. J Clin Oncol. 2013;31(6):794-810.

33. Carmona-Bayonas A, Jiménez-Fonseca P, Virizuela Echaburu J, Sánchez Cánovas M, de la Peña AF. The time has come for new models in febrile neutropenia: a practical demonstration of the inadequacy of the MASCC score. Clin Transl Oncol. 2017;19(9):1084-90.

34. Carmona-Bayonas A, Jiménez-Fonseca P. CISNE or MASCC, which predictor is really the weakest in febrile neutropenia? Eur J Intern Med. 2018;50:e33-4.

35. Clark OAC, Lyman GH, Castro AA, Clark LGO, Djulbegovic B. Colony-stimulating factors for chemotherapy-induced febrile neutropenia: a meta-analysis of randomized controlled trials. J Clin Oncol. 2005;23(18):4198-214.

36. García-Carbonero R, Mayordomo JI, Tornamira MV, López-Brea M, Rueda A, Guillem V, et al. Granulocyte colony-stimulating factor in the treatment of high-risk febrile neutropenia: a multicenter randomized trial. J Natl Cancer Inst. 2001;93(1):31-8.

37. Mercier T, Maertens J. Clinical considerations in the early treatment of invasive mould infections and disease. J Antimicrob Chemother. 2017;72(suppl_1):i29-38.

38. van Dalen EC, Mank A, Leclercq E, Mulder RL, Davies M, Kersten MJ, et al. Low bacterial diet versus control diet to prevent infection in cancer patients treated with chemotherapy causing episodes of neutropenia. Cochrane Database Syst Rev. 2016;4:CD006247.

39. Allegranzi B, Pittet D. Role of hand hygiene in healthcare-associated infection prevention. J Hosp Infect. 2009;73(4):305-15.

40. Siegel JD, Rhinehart E, Jackson M, Chiarello L. 2007 guideline for isolation precautions: preventing transmission of infectious agents in health care settings. Am J Infect Control. 2007;35(10):S65-164.

41. Carmona-Bayonas A, Gordo F, Beato C, Castaño Pérez J, Jiménez-Fonseca P, Virizuela Echaburu J, et al. Intensive care in cancer patients in the age of immunotherapy and molecular therapies: commitment of the SEOM-SEMICYUC. Med Intensiva. 2018;42(6):363-9.

42. Clinical Index of Febrile Neutropenia WebSite; 2015. http://www. iricom.es/prognostictools/Cisne/inicio.aspx. Accessed 15 Oct 2018.

43. Virizuela JA, Carratalà J, Aguado JM, Vicente D, Salavert M, Ruiz M, et al. Management of infection and febrile neutropenia in patients with solid cancer. Clin Transl Oncol. 2016;18(6):557-70.

44. Lyman GH, Kuderer NM, Crawford J, Wolff DA, Culakova E, Poniewierski MS, et al. Predicting individual risk of neutropenic complications in patients receiving cancer chemotherapy. Cancer. 2011;117(9):1917-27.

45. Baden LR, Swaminathan S, Angarone M, Blouin G, Camins BC, Casper C, et al. Prevention and treatment of cancer-related infections, Version 2.2016, NCCN Clinical Practice Guidelines in Oncology. J Natl Compr Cancer Netw. 2016;14(7):882-913.

46. Smith TJ, Khatcheressian J, Lyman GH, Ozer H, Armitage JO, Balducci L, et al. 2006 update of recommendations for the use of white blood cell growth factors: an evidence-based clinical practice guideline. J Clin Oncol. 2006;24(19):3187-205.

47. Mhaskar R, Clark OAC, Lyman G, Botrel TEA, Paladini LM, Djulbegovic B. Colony-stimulating factors for chemotherapyinduced febrile neutropenia. Cochrane Database Syst Rev. 2014;10:CD003039.

48. Crawford J, Glaspy JA, Stoller RG, Tomita DK, Vincent ME, McGuire BW, et al. Final results of a placebo-controlled study of filgrastim in small-cell lung cancer: exploration of risk factors for febrile neutropenia. Support Cancer Ther. 2005;3(1):36-46.

49. Person AK, Pettit AC, Sterling TR. Diagnosis and treatment of latent tuberculosis infection: an update. Curr Respir Care Rep. 2013;2(4):199-207. 